\title{
REGULARITY OF RIESZ MEASURES
}

\author{
P. PRINZ
}

\begin{abstract}
It is shown that Riesz measures are inner regular, i.e., each Borel set may be approximated from inside by closed sets, if the basic space is metacompact or para-Lindelöf. On the other hand, an example is given to show that local compactness is not sufficient to ensure inner regularity of Riesz measures.
\end{abstract}

1. Introduction. Regularity is an important tool to ensure the desired behaviour of measures in topological spaces and to classify them by their properties in relation to the topology (see, e.g., [4]). For instance, Radon measures are those locally finite Borel measures which are compact inner regular. It is well known that Radon measures are inner regular but not necessarily outer regular: there are standard examples of $\sigma$-finite Radon measures on separable (resp. metrisable), locally compact spaces which fail to be outer regular (cf. [5, Examples 6 and 7]). The regularisation from outside leads to the dual concept of Riesz measures, which sometimes is more adequate than that of Radon measures, e.g. in connection with the Riesz representation theorem. Of course, a Riesz measure need not be compact inner regular. But what about inner regularity? In this paper it is shown that inner regularity holds for Riesz measures in a wide class of topological spaces including the metrisable and the Lindelöf spaces. On the other hand an example shows that a locally compact space may support a Riesz measure which is not inner regular.

2. Preliminaries. Throughout this paper, all spaces will be Hausdorff. A space $X$ is called paracompact (para-Lindelöf) if each open cover has a locally finite (locally countable) open refinement. $X$ is called metacompact (meta-Lindelöf) if each open cover has a point-finite (point-countable) open refinement. Since there are paraLindelöf spaces which are not metacompact, and conversely, metacompact spaces which are not para-Lindelöf (cf. [8, Examples 63, 144]), no other than the trivial implications are valid.

Let $X$ be a fixed space and denote by $\mathcal{G}, \mathcal{F}$ and $\mathcal{K}$ the families of all open, closed and compact subsets of $X$, respectively. A Borel measure in $X$ is a nonnegative, not necessarily finite measure defined on the Borel $\sigma$-algebra $B$ of $X$. All Borel measures will be locally finite, i.e. each point has a neighbourhood of finite measure. A Borel measure $\mu$ in $X$ is called

(a) outer regular if $\mu(B)=\inf \{\mu(G): B \subset G \in \mathcal{G}\}$ for each $B \in B$;

(b) inner regular if $\mu(B)=\sup \{\mu(F): B \supset F \in \mathcal{F}\}$ for each $B \in B$;

(c) compact inner regular if $\mu(B)=\sup \{\mu(K): B \supset K \in \mathcal{K}\}$ for each $B \in B$;

(d) weakly compact inner regular if $\mu(G)=\sup \{\mu(K): G \supset K \in \mathcal{K}\}$ for each $G \in \mathcal{G}$.

Received by the editors September 10, 1984.

1980 Mathematics Subject Classification. Primary 28C15; Secondary 54D18. 
A Radon measure is a locally finite, compact inner regular Borel measure, whereas a Riesz measure is a locally finite Borel measure which is weakly compact inner regular and outer regular.

There is a natural bijection between the class of Radon measures and the class of Riesz measures (see [7, pp. 12-15]). Indeed, for each Radon measure $m$ there exists the smallest Riesz measure $M$ dominating $m ; M$ is obtained from $m$ by regularisation from outside, i.e.,

$$
M(B)=\inf \{m(G): B \subset G \in \mathcal{G}\} \text { for } B \in B .
$$

Conversely, if $M$ is a Riesz measure, then $m$ defined by

$$
m(B)=\sup \{M(K): B \supset K \in \mathcal{K}\} \quad \text { for } B \in B
$$

is the largest Radon measure dominated by $M$. The duality given by (i) and (ii) is very useful, for instance to define Riesz measures (on subspaces) via Radon measures. Clearly, two associated measures $m$ and $M$ coincide on $\mathcal{K} \cup \mathcal{G}$ and, using a standard argument, also on $\{B \in B: M(B)<\infty\}$.

A concassage of a Radon measure $m$ in $X$ is a disjoint family $C$ of nonempty compact subsets of $X$ satisfying

(a) $m(B)=\sum_{K \in C} m(K \cap B)$ for each $B \in B$;

(b) $m(K \cap G)>0$ if $K \in C, G \in \mathcal{G}$ and $K \cap G \neq \varnothing$.

Zorn's lemma provides that each Radon measure has a concassage (see, e.g., [7, p. 46, Theorem 13]). It follows directly from the definition that each open set with finite measure meets at most a countable number of sets $K \in C$. In view of the above-mentioned duality we say that $C$ is a concassage of a Riesz measure $M$ if $C$ is a concassage of the associated Radon measure $m$.

3. Main result. As will be shown in $\S 4$, there exists a Riesz measure in a locally compact space which is not inner regular. However, if the basic space $X$ has some very weak covering property then each Riesz measure in $X$ is regular.

THEOREM. Let $M$ be a Riesz measure on a Hausdorff topological space $X$. Then $M$ is inner regular if one of the following conditions is satisfied:

(a) $X$ is metacompact;

(b) $X$ is para-Lindelöf;

(c) $X$ is meta-Lindelöf and $M$ has a concassage consisting of separable subsets.

PROOF. 1. Since a Riesz measure is $\tau$-smooth, we may assume that the support of $M$ is the whole space, i.e.,

$$
M(G)>0 \text { for each } \varnothing \neq G \in \mathcal{G} .
$$

2. Now let $B$ be an arbitrary Borel subset of $X$. Clearly, it is sufficient to consider the case $m(B)<M(B)$. In particular, if $\mathcal{G}_{f}=\{G \in \mathcal{G}: M(G)<\infty\}$, then

$$
B \backslash \bigcup_{n \in \mathbf{N}} G_{n} \neq \varnothing \quad \text { for each sequence }\left(G_{n}\right)_{n \in \mathbf{N}} \text { in } \mathcal{G}_{f} \text {. }
$$

3. According to the assumptions we choose an open refinement $A$ of $\mathcal{G}_{f}$, i.e., $A$ is point-finite in case (a), locally countable in case (b) and point-countable in case (c). By Zorn's lemma there is a maximal subset $F$ of $B$ such that

$$
|F \cap U| \leq 1 \text { for each } U \in A \text {. }
$$


$F$ is uncountable. For otherwise it follows from the point-countability of $A$ that the family $A^{\prime}=\{U \in A: U \cap F \neq \varnothing\}$ is countable and hence not a cover of $B$, using (2) above. Consequently we can add a point $x \in B \backslash \bigcup \mathcal{A}^{\prime}$ to $F$ without violating (3), a contradiction to the definition of $F$.

4. Since $A$ is a cover of $X,(3)$ assures that $F$ is closed. Thus it is left to prove $M(F)=\infty$. In part 5 below we will see

$$
A_{G}=\{U \in A: M(U \cap G)>0\}
$$

is countable for each $G \in \mathcal{G}_{f}$. Hence, in view of $(1)$, the family $\{U \in A: U \cap G \neq \varnothing\}$ is countable for each $G \in \mathcal{G}_{f}$. As $F$ is uncountable, it follows by using (3) again, that $F$ is not contained in an open set of finite measure. This means $M(F)=\infty$.

5. (a) Let $A$ be point-finite and suppose that the set $A_{G}$ in (4) is uncountable for some $G \in \mathcal{G}_{f}$. Then there are $\varepsilon>0$ and $U_{n} \in \mathcal{A}_{G}, n \in \mathbf{N}$, such that $M\left(U_{n} \cap G\right) \geq \varepsilon$ for each $n \in \mathbf{N}$. This yields

$$
M\left(\limsup _{n \in \mathbf{N}}\left(U_{n} \cap G\right)\right) \geq \underset{n \in \mathbf{N}}{\limsup } M\left(U_{n} \cap G\right) \geq \varepsilon,
$$

in particular, $\lim \sup _{n \in \mathbf{N}} U_{n} \neq \varnothing$. But this contradicts the assumption on $A$, and thus $A_{G}$ is countable.

(b) Now let $A$ be locally countable. Fix a concassage $C$ of $M$, and note that the family $A_{K}=\{U \in A: U \cap K \neq \varnothing\}$ is countable for each $K \in A$. Further, each $G \in \mathcal{G}_{f}$ meets only countably many sets $K \in C$. This shows that $A_{G}$ is countable, since by the weak compact regularity of $M$ we have

$$
A_{G} \subset \bigcup\left\{A_{K}: K \cap G \neq \varnothing\right\} \text {. }
$$

(c) If $A$ is point-countable and $D$ a concassage as required in the hypotheses, then the separability of each $K \in D$ implies that the family $A_{K}=\{U \in A: U \cap K \neq \varnothing\}$ is countable for each $K \in D$. Thus, proceeding as above, the assertion (4) follows.

REMARKS. 1. The essential point of the preceding proof is the interesting fact that each non- $M$-moderated subset $A$ of $X$-i.e., $A$ admits no countable cover by open sets of finite $M$-measure-contains an uncountable, closed, discrete subset of infinite measure.

2. Since an element of a concassage satisfies the countable chain condition (CCC), the assumption on the existence of a "separable concassage" is very weak. Indeed, according to part 5 (c) of the above proof, Riesz measures are inner regular on each meta-Lindelöf space supposing

H In a compact Hausdorff CCC space each point-countable cover is countable.

The hypothesis $\mathbf{H}$ is consistent with the axioms of Zermelo-Fraenkel set theory, but incompatible with the continuum hypothesis. For a detailed discussion of $\mathbf{H}$ we refer to [6].

4. An example. Let $\omega$ and $\Omega$ denote the first infinite and the first uncountable ordinal, respectively. Identifying a given ordinal with the set of all smaller ordinals we define on $X=(\omega+1) \times \Omega$ a topology as follows: the points $(n, \alpha) \in \omega \times \Omega$ are isolated and a local base at $(\omega, \alpha) \in\{\omega\} \times \Omega$ is given by the sets

$$
\left.\left.\left.\left.\bigcup_{\beta<\gamma \leq \alpha}\right] n_{\gamma}, \omega\right] \times\{\gamma\} \quad \text { where } \beta<\alpha \text { and } n_{\gamma}<\omega \text { for each } \gamma \in\right] \beta, \alpha\right] \text {. }
$$


Clearly $X$ is Hausdorff and completely regular, for $X$ is zero-dimensional. Further $Y=\{\omega\} \times \Omega$ is a closed subset and with the induced topology homeomorphic to $\Omega$ with the usual order topology. Since $\Omega$ is countably compact but not compact, $Y$ and thus $X$ is not metacompact (e.g. [2, Theorem 5.3.1]). $X$ also fails to be metaLindelöf, which may be seen by purely topological arguments (cf. [2, Theorem 5.3.3]), but is a direct consequence of the preceding Theorem, since $X$ admits a discrete Radon measure whose associated Riesz measure is not inner regular.

Let $\left(a_{n}\right)_{n \in \omega}$ be a sequence of positive real numbers with $\sum_{n \in \omega} a_{n}<\infty$ and define a measure on $B$ by

$$
m(B)=\sum_{\alpha \in \Omega} \sum_{n \in B^{\alpha}} a_{n} \text { for } B \in B
$$

where $B^{\alpha}=B \cap(\omega \times\{\alpha\})$. As $\left(a_{n}\right)_{n \in \omega}$ is summable, it follows directly from the definitions that $m$ is locally finite and therefore a Radon measure in $X$. Let $M$ be the Riesz measure associated with $m$. Then $M$ is locally finite, too, which yields

$$
M(B)=0 \text { for each bounded subset } B \text { of } Y .
$$

On the other hand, if $G$ is an open subset of $X$ such that $G \cap Y$ is unbounded then we have $m(G)=\infty$, since by definition $G$ contains uncountably many points of $\omega \times \Omega$, each of which has positive measure. This, together with the outer regularity, implies

$$
M(B)=\infty \quad \text { if } B \in B \text { with } B \cap Y \text { unbounded. }
$$

Now let $U$ be an open subset of $\Omega$ such that $U$ and $\Omega \backslash U$ are unbounded, e.g., $U=\{\alpha+1: \alpha \in \Omega\}$. Since two unbounded closed subsets of $\Omega$ have a nonvoid intersection, $U$ contains no unbounded closed subset and the same holds true for $B=\{\omega\} \times U$ in $Y$. Consequently, $B$ is a Borel set in $X$ satisfying $M(B)=\infty$ and $M(F)=0$ for each closed subset $F$ of $B$.

REMARKS. 1. The space $X$ of the preceding example is not locally compact, since a basic neighbourhood of $(\omega, \alpha)$ is closed and may be covered by the disjoint open sets $\left\{n_{\gamma}+1\right\}, \beta<\gamma \leq \alpha$, and $\left.\left.\bigcup_{\beta<\gamma \leq \alpha}\right] n_{\gamma}+1, \omega\right] \times\{\gamma\}$. To get a nonregular Riesz measure in a locally compact space, we use a general method of embedding suggested by Fremlin $[\mathbf{3}, 2 \mathrm{~J}]$. As $X$ is completely regular, we regard $X$ as a subspace of its Stone-Cech compactification $\beta X$. Set

$$
\hat{X}=\bigcup\{G: G \subset \beta X \text { open and } M(G \cap X)<\infty\} .
$$

Since $\hat{X}$ is an open subset of $\beta X$ and $M$ is locally finite, $\hat{X}$ is locally compact and $X \subset \hat{X}$. Define $\hat{M}$ in $\hat{X}$ by

$$
\begin{aligned}
& \hat{M}(G)=M(G \cap X) \text { for an open set } G \subset \hat{X}, \\
& \hat{M}(B)=\inf \{\hat{M}(G): G \subset \hat{X} \text { open and } B \subset G\} \text { for a Borel set } B \subset \hat{X} .
\end{aligned}
$$

Clearly $M(B)=\hat{M}^{*}(B)$ holds for each Borel set $B$ of $X$. Moreover, $\hat{M}$ is a Riesz measure in $\hat{X}$, since $\hat{M}$ is the associated measure of the Radon measure $\hat{m}$ in $\hat{X}$ defined by $\hat{m}(B)=m(B \cap X)$ for a Borel set $B \subset \hat{X}$. Like $M, \hat{M}$ is not inner regular. To see this, it is enough to show that $Y$ is a Borel set in $\hat{X}$. Indeed, $Y$ is even closed in $\hat{X}$. Let $U$ be an open neighbourhood of a point in $\hat{X} \backslash Y$ with $\hat{M}(U)<\infty$. Then $U$ intersects $Y$ in a bounded set only, and consequently $U \backslash Y=U \backslash I$ for a 
suitable bounded closed interval $I$ in $Y$. But $I$ is compact in $Y$ and hence closed in $\hat{X}$. This shows that $U \backslash Y$ is open in $\hat{X}$.

2. The author does not know of a separable, locally compact space which supports a nonregular Riesz measure. It also remains open whether in the above Theorem the assumption on the covering properties of $X$ may be weakend, e.g. to $\theta$-refinable $($ see $[\mathbf{1}])$.

\section{REFERENCES}

1. H. R. Bennett and D. J. Lutzer, A note on weak $\theta$-refinability, General Topology and Appl. 2 (1972), 49-54.

2. R. Engelking, Outline of general topology, North-Holland, Amsterdam, 1968.

3. D. H. Fremlin, Topological measure spaces: two counterexamples, Math. Proc. Cambridge Philos. Soc. 78 (1975), 95-106.

4. R. J. Gardner, The regularity of Borel measures, (Proc. Conf. Measure Theory, Oberwolfach, 1981), Lecture Notes in Math., vol. 945, 1982, pp. 42-100.

5. G. Gruenhage and W. F. Pfeffer, When inner regularity of Borel measures implies regularity, J. London Math. Soc. (2) 17 (1978), 165-171.

6. K. Kunen and F. D. Tall, Between Martin's axiom and Souslin's hypothesis, Fund. Math. 102 (1979), 173-181.

7. L. Schwartz, Radon ineasures on arbitrary topological spaces and cylindrical measures, Oxford Univ. Press, London, 1973.

8. L. A. Steen and J. A. Seebach, Counterexamples in topology, 2nd ed., Springer-Verlag, Berlin-Heidelberg-New York, 1978.

MAThematisches Institut DER Universität MÜNChen, Theresienstasse 39, D8000 MÜNCHEN 2, FEDERAL REPUBLIC OF GERMANY 